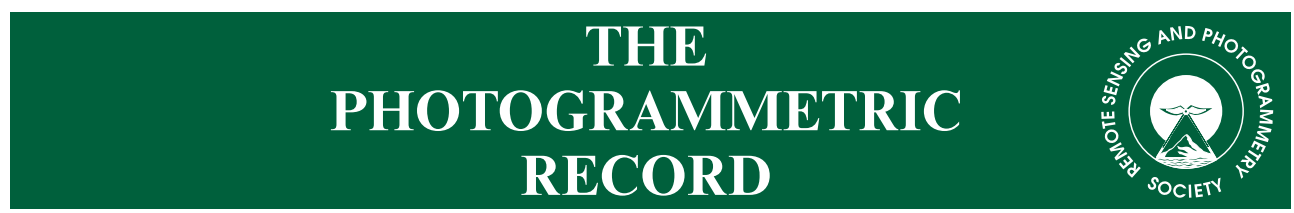

The Photogrammetric Record 30(152): 339-362 (December 2015)

DOI: $10.1111 /$ phor. 12122

\title{
CREATION OF PARAMETRIC BIM OBJECTS FROM POINT CLOUDS USING NURBS
}

\author{
L. BARAZZeTti (luigi.barazzetti@polimi.it) \\ F. BANFI (fabrizio.banfi@polimi.it) \\ R. BRUMANA (raffaella.brumana@polimi.it) \\ M. Previtali (mattia.previtali@polimi.it) \\ Politecnico di Milano, Milan, Italy
}

\begin{abstract}
This paper presents an innovative procedure to create parametric building information modelling (BIM) objects from point clouds of complex architectural features. BIM technology requires an advanced parametric representation of the geometry involving spatial relationships, constraints and material properties. The aim of the procedure is a BIM-based reconstruction methodology that preserves the level of detail encapsulated in photogrammetric and laser-scanning point clouds, and relies on non-uniform rational basis splines (NURBS) curves. The particular case of architectural objects with irregular shapes is addressed due to the lack of commercial BIM software able to handle such buildings. An actual case study made up of 7.5 billion points is discussed to demonstrate the use of the proposed procedure with huge point-cloud datasets.
\end{abstract}

KEYWORDS: BIM, building information modelling, interoperability, NURBS, point cloud

\section{INTRODUCTION}

BUILDING INFORMATION MODELLING (BIM) is one the most promising technologies to increase productivity in the architectural, engineering and construction (AEC) industries. Increasingly, $\mathrm{BIM}$ is becoming the standard for the design, construction and management of new buildings, notwithstanding some resistance from certain specialists (Eastman et al., 2008; Fai et al., 2013).

The growing interest in BIM is confirmed by the development of numerous commercial software (examples include Revit, ArchiCAD, Vectorworks, Bentley Systems, Allplan, Tekla Structures and RIB iTWO) that take into consideration the different requirements of various specialists, such as architects, engineers, designers, planners, construction and facility managers, involved in the construction process (Azhar, 2011; Azhar et al., 2012). BIM can be implemented as a common environment able to manage the different stages of a project: planning, design, construction and management. Practical case studies have proved that the use of a common BIM platform leads to an incremental rise in field productivity of up to $40 \%$ (Chelson, 2010). 
BIM is an evolution of computer-aided design (CAD) systems. Historically, hand drawings using boards and manual drafting were progressively substituted by $2 \mathrm{D}$ and $3 \mathrm{D}$ vector-based CAD representations (starting in the 1970s and 1980s). This resulted in an immediate advantage in terms of project productivity, with a consistent reduction of design errors and omissions. However, the creation of multiple CAD drawings (plans, elevations, sections, 3D models and so on) with multiple revisions (independently carried out by different specialists) was often the cause of unsynchronised and inconsistent project versions.

The BIM paradigm moves the traditional design concept towards reducing problems caused by the lack of communication between specialists who can take advantage of new interoperability standards. BIM relies on a 3D model of the structure with additional parametric geometry so that objects can be modified without redrawing. BIM is much more than a crude 3D representation made up of static shapes with fixed geometry (Oreni et al., 2014). It is an object-oriented database of the building with an improved coordination of construction documents where geometry, spatial relationships and the properties of building components are structured. Construction elements become intelligent objects containing information (for example, materials and layers) integrated with their physical dimensions. Intelligence is added by the integration of building attributes that describe the elements of nested objects which have a relationship to one another (Kensek and Noble, 2014).

The growing interest in BIM technology has a direct impact on the requirements of image- and range-based reconstructions. Photogrammetry and laser scanning are powerful tools that capture huge quantities of $3 \mathrm{D}$ metric information about an object, especially with the recent developments in image matching and orientation algorithms (Barazzetti et al., 2010; Jazayeri and Fraser, 2010; Gruen, 2012; Remondino et al., 2014). However, after considering the main innovations coming from building information modelling, additional issues arise. The question behind the current work is: "How can one turn point clouds into rigorous BIM?"

Multiple factors should be taken into account to answer the question. In addition, very little research has been undertaken to understand the potential of BIM for heritage buildings (Fai et al., 2011). Most BIM software have families of objects relating only to modern constructions, and the reconstruction of complex shapes seems a challenging task.

A further important consideration must be pointed out before developing this topic: 3D models that contain only 3D (metric) data are not BIM models (Arayci, 2008). This means that a model generated from point clouds is not a BIM unless (1) parametric intelligence, (2) relationships and (3) attributes are added. Parametric intelligence means that an object (such as walls, doors and windows) can be encapsulated into the project and modified with a simple set-up of its dimensions in a project database. Different objects are consistent (for example, a door fits into a wall) in order to remove redundant information and project errors; a change in a specific object should also modify the objects with which it has a direct interaction. In contrast, attributes are additional features that describe the properties of structural elements, such as component materials and energy data.

As BIM requires object-oriented parametric modelling techniques, methods that try to reconstruct the shape with a direct interpolation of point clouds cannot produce BIM models. The logic of construction of the elements (how the building is assembled) and material properties cannot be neglected. This information comes at a cost and is the sort of knowledge that reveals that interconnections between component construction elements are mandatory in order to obtain a set of consistent objects. This is probably one of the most complicated areas for the automated generation of intelligent models: BIM requires the 
identification of single elements and their mutual relationships in order to create a model composed of a large number of simple parts (Eastman et al., 2008).

On the other hand, it is clear that the information given by point clouds is a powerful tool to initialise the geometry of BIM. High-resolution and accurate point clouds can reveal the external shape and its irregularities. Simple and regular objects (such as straight walls) can be modelled with the families available in most BIM packages that were mainly developed for new buildings. Most software vendors are adapting their software to import and visualise point clouds to obtain a direct link between geometry, families of objects, parametric intelligence and attributes.

However, the geometric complexity that can be captured by laser-scanner and photogrammetric point clouds leads to another problem. It is very difficult to reconstruct correctly the shape with the predefined elements developed for modern and regular buildings. This problem becomes even more intricate for architectural objects that would require excessive simplification of irregular elements (such as vaults or arches), with a concomitant level of the detail resulting in almost useless models for restoration and conservation.

In the technical literature, some examples of complete BIM derived from point clouds and integrated in specific BIM packages (mainly Autodesk Revit or ArchiCAD) were discussed in Murphy et al. (2009, 2013), Fai et al. (2011), Oreni et al. (2012, 2014), Brumana et al. (2013), Baik et al. (2014), Fai and Rafeiro (2014), Scianna et al. (2014), Barazzetti et al. (2015), Dore et al. (2015) and Quattrini et al. (2015). The analysis of complex shapes was a common major challenge, for which different strategies (such as implementation of new algorithms for object generation, creation of new families or excessive simplification) were adopted. It is important to underline that the use of a commercial BIM package is mandatory to fulfil basic BIM requirements, avoiding traditional 3D models generated in pure modelling packages (such as Maya, Rhinoceros and 3D Studio Max) that do not fulfil BIM requirements because of their lack of parametric modelling tools, spatial relationships and attributes (Arayci, 2008; Remondino, 2011).

This paper presents a new procedure for the generation of accurate BIM of complicated objects, starting with dense point clouds generated with laser scanning or photogrammetric techniques. The objects obtained have parametric intelligence and follow the original shape of the construction elements, as well as their logic of construction. Attributes can be added with respect to the requirements of BIM projects.

One of the aims of this work is to prove that point clouds remain the most complete and detailed solution for capturing building geometry. Point clouds can be then converted into intelligent BIM objects with a new strategy that generates parametric geometry and relationships from a set of non-uniform rational basis splines (NURBS) curves and surfaces.

\section{Developed BIM Procedure for Complex Objects}

Most 3D modelling projects are based on procedures that are able to generate surfaces with the interpolation of the point cloud using a mesh-based algorithm. On the other hand, BIM technology requires a parametric representation of geometry and not simply a static shape. The procedure proposed in this paper exploits innovative concepts and procedures for BIM generation which preserve the metric accuracy captured by laser-scanner and photogrammetric point clouds.

The workflow is visually illustrated in Fig. 1: the laser scan of an irregular vault was turned into advanced BIM elements with the parametrisation of the vault thickness. The vault becomes an "intelligent object" with a dynamic shape and material information. 

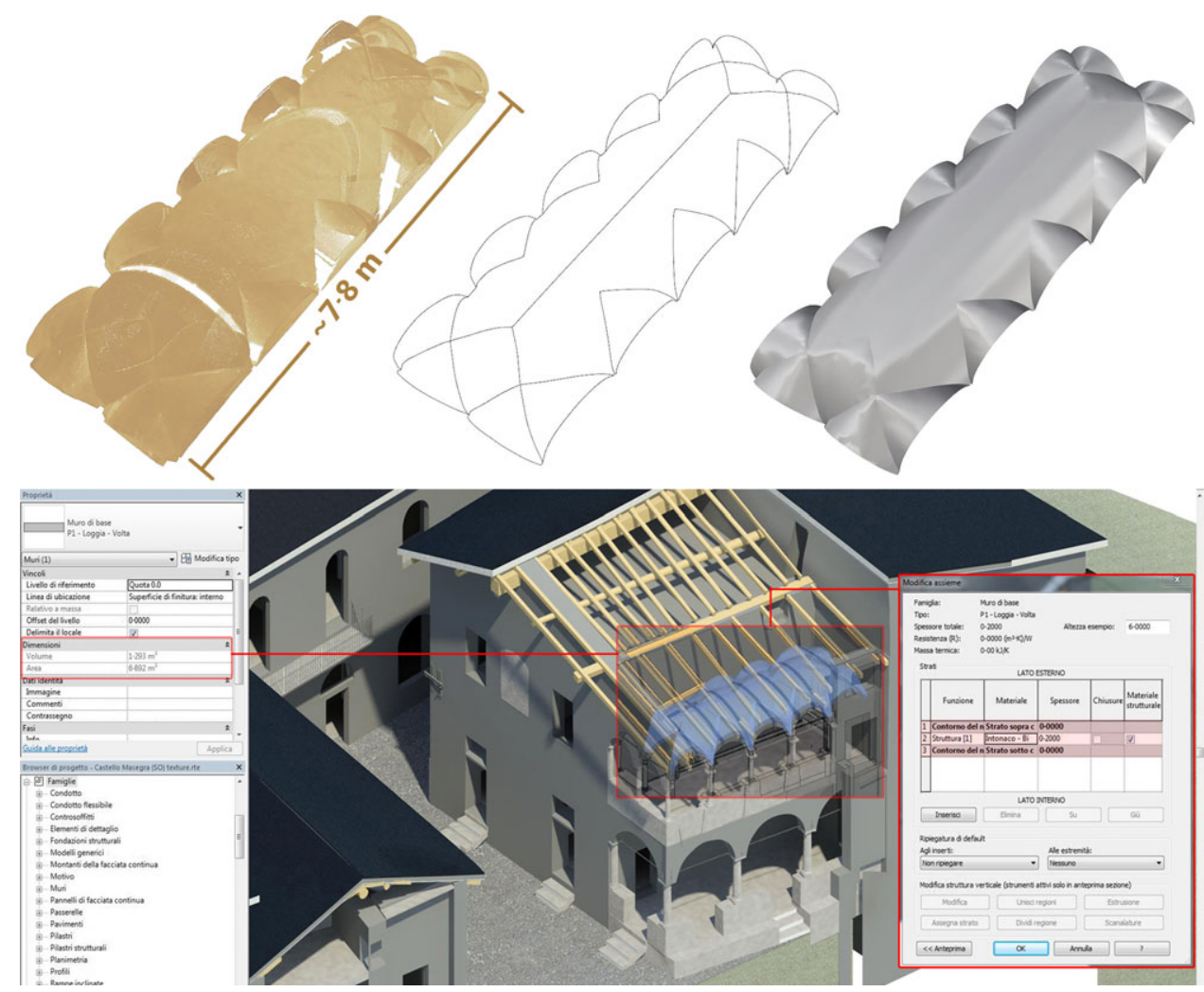

FIG. 1. Schematic workflow of the proposed approach for BIM generation. NURBS curves and surfaces are fitted to point clouds by following the discontinuity lines of construction elements; parametrisation is then carried out to transform the surfaces into an intelligent object.

The parametric modelling strategy is a semi-automated methodology based on NURBS (Piegl and Tiller, 1997) using the following four steps:

(1) NURBS curves are used to detect the discontinuity lines of the different structural elements (manual or semi-automated approach);

(2) an (optional) NURBS curve network can be generated to densify the discontinuity lines (manual or semi-automated approach);

(3) NURBS surfaces are interpolated using NURBS curves and the point cloud (automated approach);

(4) the surface is turned into a BIM object with parametric intelligence, spatial relationships and attributes (automated approach for the geometric part, the inclusion of attributes is carried out by an expert operator).

In the proposed workflow the identification of the different architectural elements is required in order to correctly replicate the logic of construction (how the building was actually constructed) integrated with spatial relationships and attributes (Hichri et al., 2013).

Real examples derived from a complex case study are illustrated and discussed to prove that the proposed reconstruction approach for irregular objects is feasible in real projects. 
The reconstructed elements can be incorporated into commercial BIM packages for further analysis involving different specialists (architects, engineers, geologists, archaeologists, historians, restorers, constructors and so on), preserving the need for interoperable solutions.

The chosen case study is Castel Masegra (Fig. 2) in Sondrio (northern Italy), which was surveyed with photogrammetric and laser-scanning techniques. A laser-scanner point cloud made up of 7.5 billion points was acquired with a Faro Focus 3D laser scanner. Overall, 176 scans were registered with a precision better than $\pm 3 \mathrm{~mm}$ by means of spherical and chequerboard targets. Laser targets were also incorporated into a robust geodetic network of 68 stations that provides a stable and accurate reference system using a Leica TS30 total station.

Additional metric information was collected with photogrammetric techniques and calibrated cameras (Cronk et al., 2006) with different lenses. Images were used to create additional point clouds and orthophotos of the external façades, internal walls and vaults. This was useful to inspect the layers of the walls and add more information in the BIM. The inspection of the roof was carried out with an unmanned aerial vehicle (UAV) platform, namely, an AscTec Falcon 8 (Barazzetti et al., 2014).

It is well known that the combined use of different recording tools and techniques is mandatory for the reconstruction of complex sites (Riveiro et al., 2008; Cabaleiro et al., 2014). Image-based (Remondino and El-Hakim, 2006) and range-based (Lerma et al., 2014) techniques are powerful solutions for obtaining accurate geometric information of complex sites (Guidi et al., 2009; Fassi et al., 2011). Subsequent data processing must take into consideration the needs of the specialists involved in the project to arrive at a common outcome of the work. From this point of view, BIM is an innovative tool in the restoration and conservation activities of the castle.

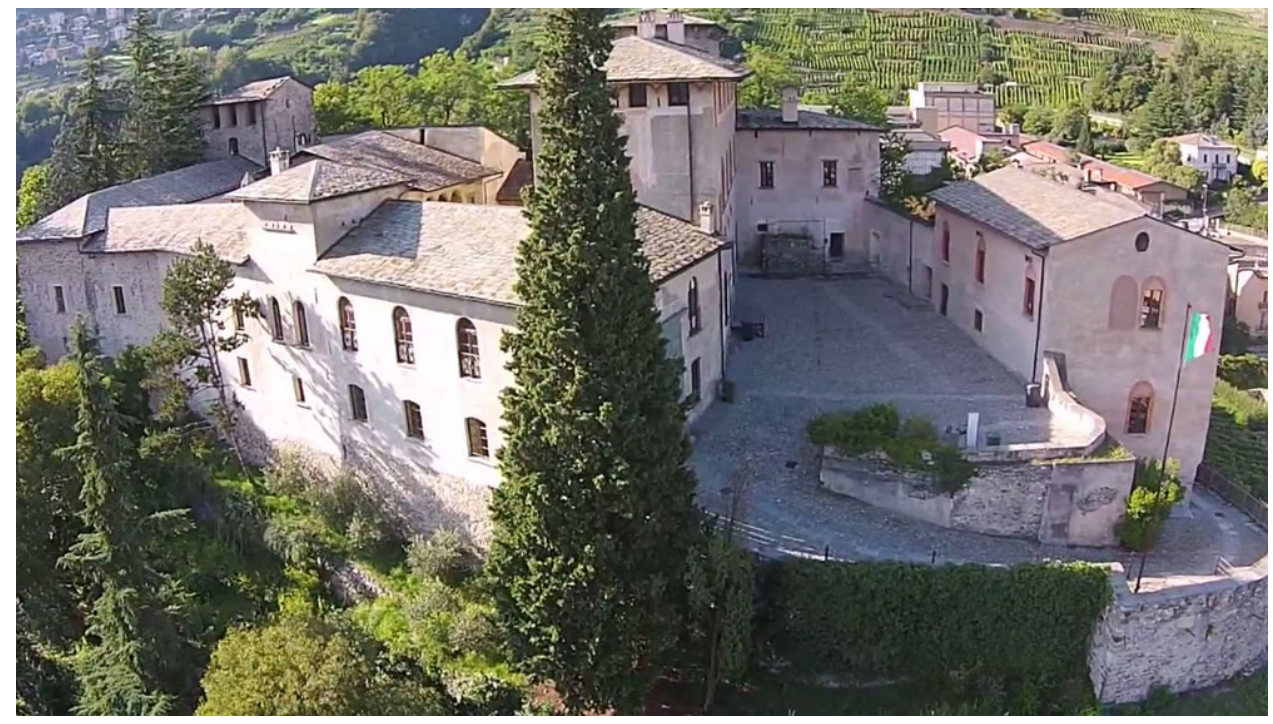

FIG. 2. Castel Masegra in Sondrio, Italy, surveyed with laser scanning and photogrammetric techniques for BIM generation. 
Selected images of the geodetic network and the laser point clouds, as turned into the final parametric BIM, are shown in Fig. 3. The elements were generated by taking into consideration: the geometric complexity and the materials; the state of conservation; the structural connections; and the presence of cracks, decorations and frescos. This illustrates how different specialists with different backgrounds are needed in a complete restoration project.

The final BIM file (.rte format) was larger than 470 megabytes and is made up of several families (for example, wall, roof, ceiling, stairs, beam, railing, column, door, generic object, window). Overall, the model consisted of 6310 elements. Simple objects were modelled directly in Autodesk Revit, whereas complex shapes required the proposed procedure due to the lack of flexible modelling procedures in commercial software. This indicates that much further research work is still needed to implement BIM-based
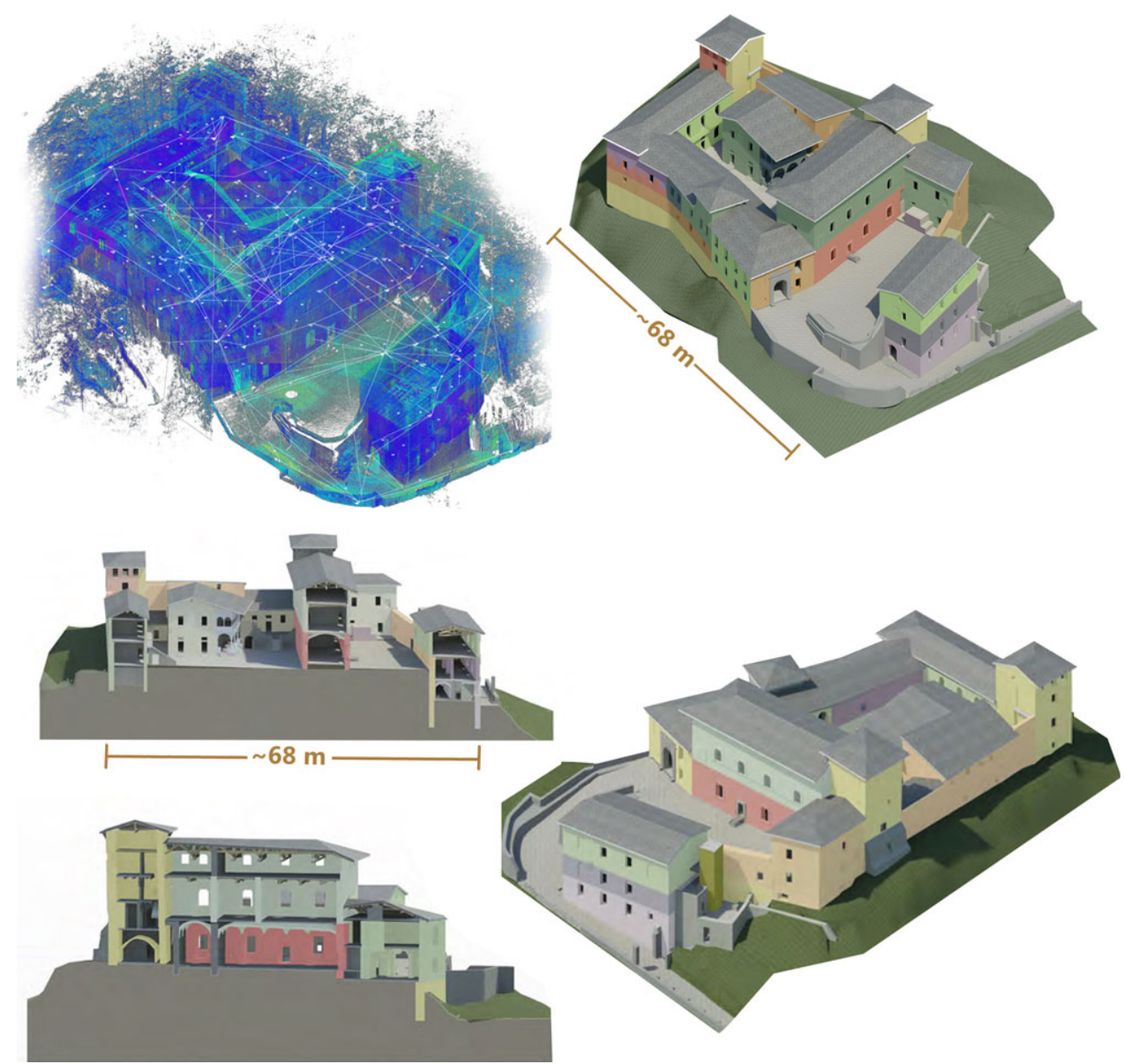

FIG. 3. Laser scanning point clouds ( 7.5 billion points) and geodetic network used to generate the BIM of Castel Masegra, which is made up of intelligent objects with geometric parametrisation, relationships and attributes. Colours correspond to different construction stages based on historical research. 
algorithms able to provide a detailed reconstruction for architectural objects with complex shapes.

The work carried out in the commercial package Autodesk Revit consists of the digital modelling of parts that can be reasonably approximated with standard Revit families (for instance, floors and ceilings are quite regular after the inspection of laser scans). Complex objects (such as vaults) were instead reconstructed with the new procedure based on NURBS curves and surfaces. Material and layer information was included in the attribute table, as well as the construction stages from the historical research. Different parametrisation levels were used for different objects, including a partial or full parametrisation depending on functional requirements.

A schematic visualisation for one of the buildings of the castle is shown in Fig. 4. Simple elements (modelled in Autodesk Revit) are combined with complex vaults reconstructed with the implemented procedure, as described in the next sections. The final result is a complete BIM solution for 3D modelling that exploits point clouds for both simple and advanced shapes. The use of a commercial package like Autodesk Revit as a final choice ensures that the model is also used by specialists that are not expert in the field of photogrammetry. Specialist ad hoc software is not necessary, meaning that the photogrammetric results can be used by a wide user community interested in BIM for productive work.

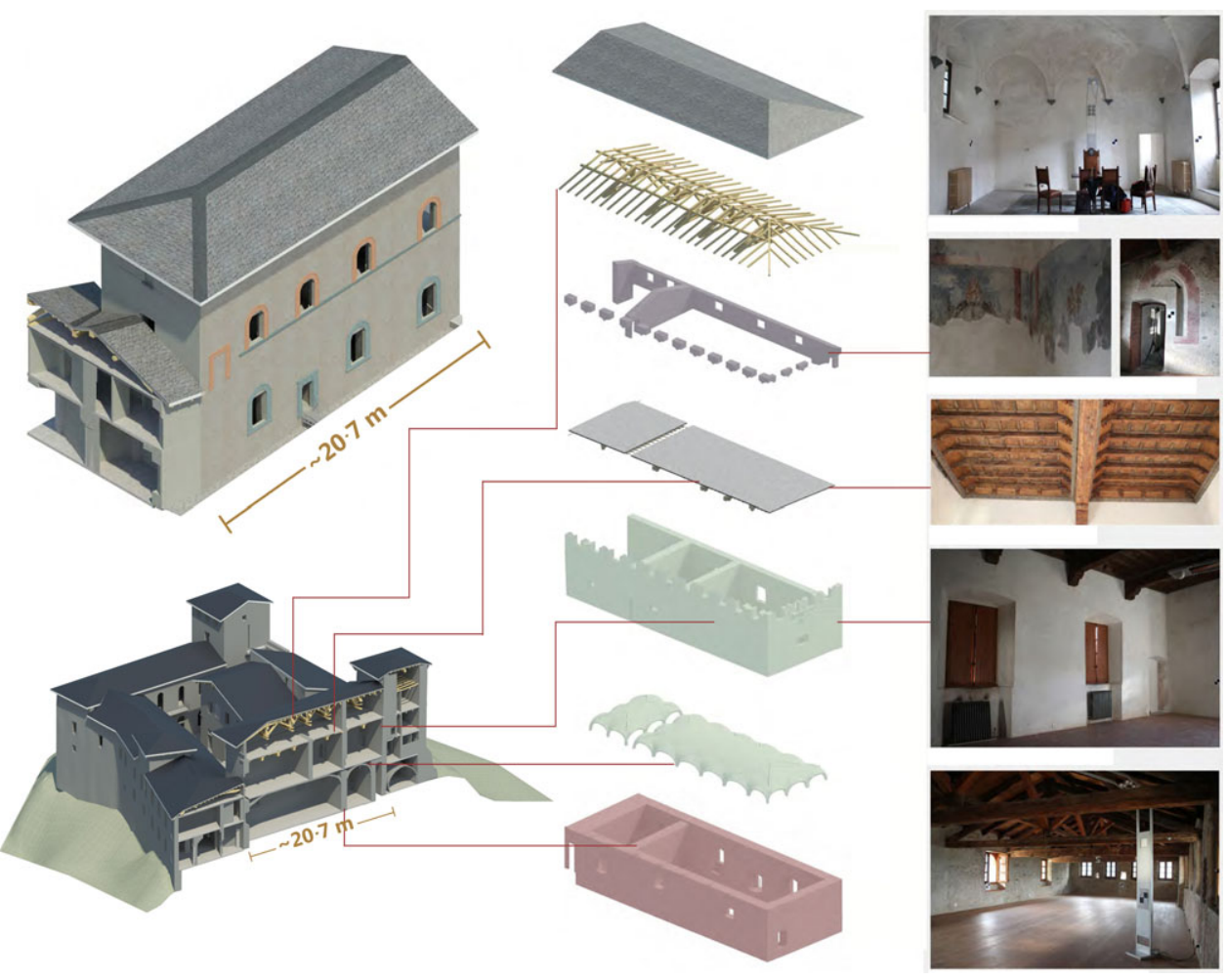

FIG. 4. The final BIM can be handled in Revit. Simple objects (for example, trusses) were directly modelled with commercial software, whereas complex objects (such as vaults) were reconstructed with the developed procedure and then imported into Revit. 


\section{Generation of a NURbS Curve Network from Point Clouds}

NURBS are powerful tools for the reconstruction of both simple shapes and complex objects (Piegl, 1991). NURBS are mathematical functions defined in parametric form and can be interactively manipulated by changing the position of control points (Farin, 1992, 1996; Hsu et al., 1992). Manual modelling via NURBS is feasible with an easily understood geometric interpretation offered by several commercial packages (examples include Ashlar-Vellum CAD, Blender, EvoluteTools PRO, Hexagon, Maya, Mol, Nurbana 3D and Rhinoceros).

The proposed procedure for BIM generation is based on NURBS curves derived from the point cloud. Curves are generated by taking into account the logic of the construction of the building, which is manually divided into its constructive elements. The manual generation of the discontinuity lines of the construction elements provides a rigorous separation based on the components of the building. Point clouds are a powerful starting point for an accurate reconstruction of the external layer of the different elements. On the other hand, this means that only the parts visible in the point clouds can be accurately reconstructed. Thus, laserscanning and photogrammetric techniques can reveal only the external surfaces that are just a part of the whole reconstruction process. In fact, the final BIM must incorporate volumetric information (such as wall thickness), material properties and the organisation of structural elements in a building database, which cannot be neglected in BIM projects.

In the proposed methodology, 3D NURBS curves are used to initialise the reconstruction of the different structural elements. NURBS curves form the skeleton of the proposed reconstruction approach, not only for a preliminary separation of the constructive elements, but also for the ability to highlight local anomalies such as verticality issues or variations in thickness. In some cases, a set of additional 3D curves can be generated inside the discontinuity lines to form a rigid curve network in space that allows a more accurate reconstruction along predefined directions.

The implemented strategy uses the point clouds to drive the generation of NURBS curves in space. A subset of the original point clouds can be extracted along predefined directions given by cutting planes set by the user. Cutting planes can be intended as cross sections through the point clouds and are useful to simplify the $3 \mathrm{D}$ reconstruction process towards a $2 \mathrm{D}$ analysis based on a plane arbitrarily oriented in space.

Given a set of $n+1$ control points $\mathrm{P}_{0}, \ldots, \mathrm{P}_{n}$ a NURBS curve $\mathrm{C}(u)$ of degree $p$ is defined by (Piegl, 1989a):

$$
\mathrm{C}(u)=\frac{\sum_{i=0}^{n} N_{i, p}(u) w_{i} \mathrm{P}_{i}}{\sum_{i=0}^{n} N_{i, p}(u) w_{i}}
$$

where $w_{i}$ are the weights and $N_{i, p}(u)$ are the $p$ th-degree B-spline basis functions defined on the knot vector:

$$
U=\left\{0, \ldots, 0, u_{p+1}, \ldots, u_{m-p-1}, 1, \ldots, 1\right\}
$$

Here $U$ is a non-decreasing sequence of real numbers whose elements $u_{i}$ are termed knots. The $i$ th B-spline basis function $N_{i, p}(u)$ has the recursive Cox-de Boor form (Cox, 1972):

$$
\begin{aligned}
& N_{i, 0}(u)= \begin{cases}1 & \text { if } u_{i} \leq u \leq u_{i+1} \\
0 & \text { otherwise }\end{cases} \\
& N_{i, p}(u)=\frac{u-u_{i}}{u_{i+p}-u_{i}} N_{i, p-1}(u)+\frac{u_{i+p+1}-u}{u_{i+p+1}-u_{i+1}} N_{i+1, p-1}(u) .
\end{aligned}
$$


The use of the rational basis function $\left\{R_{i, p}(u)\right\}$ defined as:

$$
R_{i, p}(u)=\frac{N_{i, p}(u) w_{i}}{\sum_{j=0}^{n} N_{j, p}(u) w_{j}}
$$

provides the compact form:

$$
\mathrm{C}(u)=\sum_{i=0}^{n} R_{i, p}(u) \mathrm{P}_{i}
$$

As already mentioned, the generation of discontinuity lines is carried out by setting specific cutting directions in space which are able to extract thin point-cloud slices. A pointcloud slice must have a limited thickness (for example, 1 to $2 \mathrm{~cm}$ ) so that its points can be assumed to be aligned. The use of multiple slices allows numerous discontinuity lines to be highlighted for the generation of complex objects. In particular, multiple horizontal cutting planes are useful for inspecting the thickness of walls and their structural anomalies, such as non-vertical or tilted walls, whereas vertical slices can initialise the generation of cross sections. Multiple horizontal, vertical and tilted slices are needed to model complex objects such as vaults.

NURBS curves can be estimated from the point clouds with manual or semi-automated measurements, depending on the complexity of their shape. The manual identification of point-cloud slices (cutting planes needed to subdivide the building into construction elements) requires an expert operator able to interpret the building. In the case of manual measurements, the user can interactively reconstruct the curve by picking some points of the slice. The degree of the curve must be set to 1 for straight lines; degrees 2 and 3 provide for an accurate representation of irregular lines.

Automation can be achieved by selecting only the start and end points of the discontinuity lines. Given a subset of equally spaced points $\mathrm{Q}_{k}(k=0, \ldots, n)$ in the pointcloud slice (the threshold for point separation is set by the user as a distance in space), which defines the points where the curve will be interpolated, the estimation of the NURBS curve requires points of an additional knot vector $U=\left\{u_{0}, \ldots, u_{m}\right\}$. Next, a $(n+1) \times(n+1)$ system of linear equations can be written as (Piegl and Tiller, 1997):

$$
\mathrm{Q}_{k}=\mathrm{C}\left(\bar{u}_{k}\right)=\sum_{i=0}^{n} N_{i, p}\left(\bar{u}_{k}\right) \mathrm{P}_{i}
$$

where the $n+1$ control points $\mathrm{P}_{i}$ are the unknowns and the parameter value $\bar{u}_{k}$ is assigned with the chord length method (Piegl and Tiller, 1997; Yeh and Hsu, 2002):

$$
\bar{u}_{k}=\bar{u}_{k-1}+\frac{\left|\mathrm{Q}_{k}-\mathrm{Q}_{k-1}\right|}{\sum_{k=1}^{n}\left|\mathrm{Q}_{k}-\mathrm{Q}_{k-1}\right|} .
$$

Some examples of three-dimensional NURBS curves used to model the discontinuity lines of complex shapes in the vaults of Castel Masegra are shown in Fig. 5. The proposed approach can be intended as a semi-automated solution where: (a) the expert operator identifies the elements and its discontinuity with some point-cloud slices; (b) the start point and end point are interactively chosen; and (c) the algorithm uses the point cloud to generate the curve. 

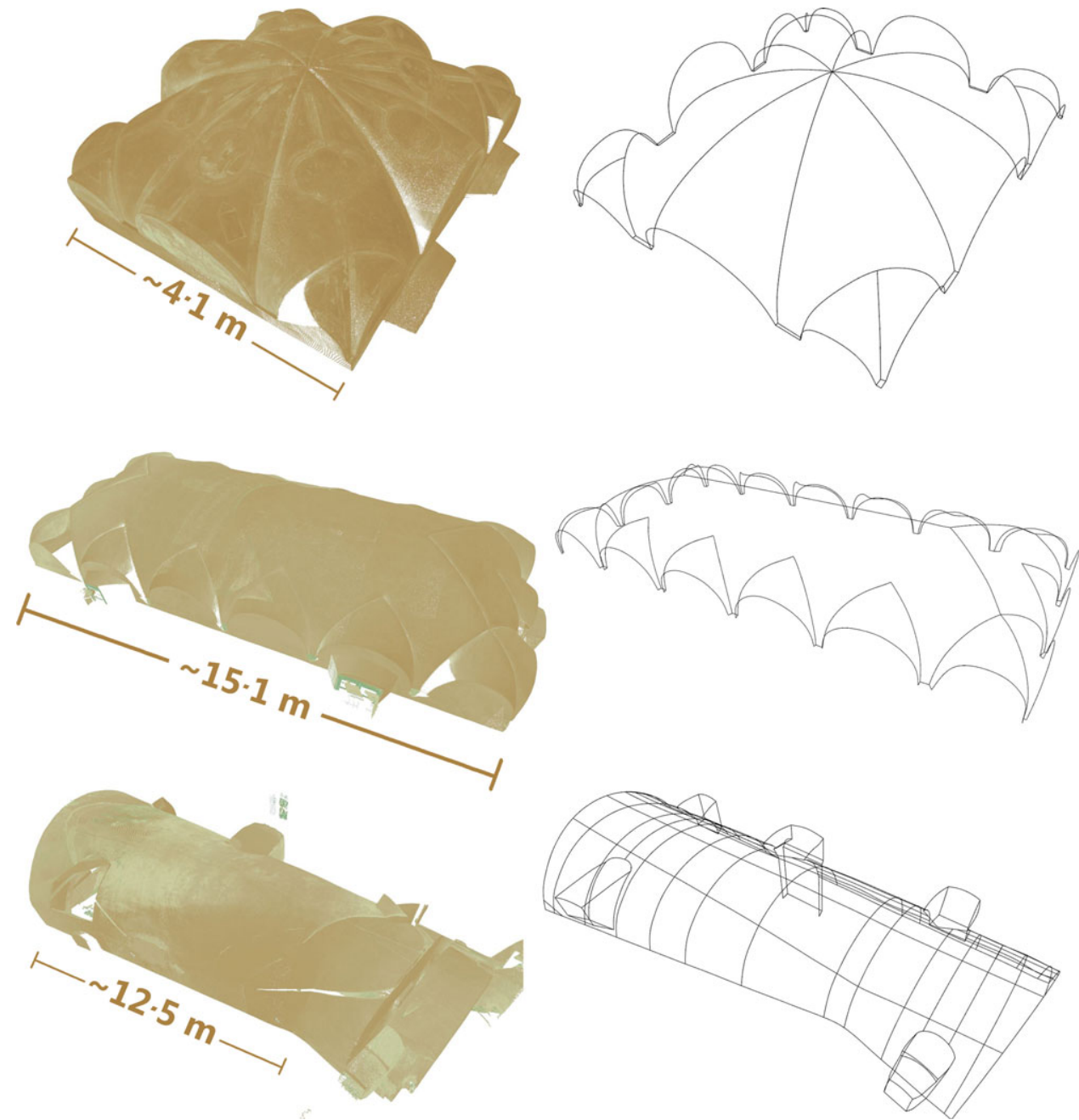

FIG. 5. Three vaults of Castel Masegra modelled with NURBS curves. Point clouds are shown on the left, whereas the generated lines on the right are based on the logic of construction of the vaults. Top: the umbrella vault in the dovecote tower. Middle: the vault with lunettes in the reception hall. Bottom: the barrel vault in the cellar.

One of the advantages of the proposed procedure is the opportunity to use the same points as constraints for multiple profiles so that there is a perfect intersection of multiple NURBS curves. In some cases, the automated selection of the subset of points $\mathrm{Q}_{k}$ is a complex task. Indeed, fully automated modelling is still not feasible, not only for complex architectural objects, but also for the structural interpretation of the building, which is a fundamental requisite for BIM. In addition, occlusions are inevitable in the case of actual projects and still require manual measurements. 


\section{Generation of NURBS Surfaces}

Three-dimensional NURBS curves provide the geometric constraints which are able to initialise the reconstruction of external surfaces of final BIM objects. In this section, the reconstruction of the visible layer (the one surveyed by point clouds) of the architectural element is described, whereas the next section will address the problem of parametric building information modelling (the internal layers and material information).

As mentioned, range- or image-based point clouds provide the shape of the external layer of the elements. They allow a digital model to be generated, but only of the outer surfaces. This incomplete reconstruction in BIM projects requires a volumetric object representation. The typical case is the vault, for which intrados can be surveyed, whereas the extrados requires a parametric BIM representation to generate a dynamic model that encapsulates the thickness so that the vault can be modified without redrawing.

The reconstruction of the surfaces surveyed with photogrammetric techniques is carried out with the previously extracted NURBS curves and a new NURBS surface $\mathrm{S}(u, v)$ of degree $(p, q)$ in the directions $(u, v)$, as defined by (Piegl, 1989b):

$$
\mathrm{S}(u, v)=\frac{\sum_{i=0}^{n} \sum_{j=0}^{m} N_{i, p}(u) N_{j, q}(v) w_{i, j} \mathrm{P}_{i, j}}{\sum_{i=0}^{n} \sum_{j=0}^{m} N_{i, p}(u) N_{j, q}(v) w_{i, j}}
$$

where $w_{i, j}$ are the weights, and $N_{i, p}(u)$ and $N_{j, q}(v)$ are the B-spline basis functions defined on the knot vectors:

$$
\begin{aligned}
U & =\left\{0, \ldots, 0, u_{p+1}, \ldots, u_{r-p-1}, 1, \ldots, 1\right\} \\
V & =\left\{0, \ldots, 0, v_{q+1}, \ldots, v_{s-q-1}, 1, \ldots, 1\right\}
\end{aligned}
$$

and

$$
r=n+p+1, \quad s=m+q+1 .
$$

The introduction of piecewise rational basis functions $R_{i, j}(u, v)$ defined as:

$$
R_{i, j}(u, v)=\frac{N_{i, p}(u) N_{j, q}(v) w_{i, j}}{\sum_{k=0}^{n} \sum_{l=0}^{m} N_{k, p}(u) N_{l, q}(v) w_{k, l}}
$$

provides the compact form:

$$
\mathrm{S}(u, v)=\sum_{i=0}^{n} \sum_{j=0}^{m} R_{i, j}(u, v) \mathrm{P}_{i, j}
$$

NURBS surfaces can be estimated from one, two or a set of curves in space that are used as geometric constraints for surface interpolation. Although NURBS surfaces can be directly fitted to an unorganised point cloud, the final representation of sharp features with well-defined edges is usually very poor. The use of a preliminary set of curves for the generation of the surface is a more reliable choice and separates the different objects.

After the generation of the discontinuity lines with NURBS curves, the whole surface is modelled with an interpolation of the curves through one or more surfaces. The curve network can be precisely interpolated if it satisfies particular geometric constraints, whereas 
an approximation is mandatory when a precise solution is mathematically impossible. In the latter case, the curve network should be interpolated as closely as possible so that the distance between the curves, the point clouds and the final surface is minimal.

The first case illustrated here allows strong control of the interpolated surface. If the curves in one direction cross all curves in the other direction (self-intersections between curves in the same direction are not allowed) the surface $\mathrm{S}(u, v)$ can be estimated with the elegant mathematical solution developed by Gordon (1993), obtaining a surface that interpolates the curves in space so that $\mathrm{C}_{k}(u)=\mathrm{S}\left(u, v_{k}\right)(0 \leq k \leq K)$ and $\mathrm{C}_{l}(v)=\mathrm{S}\left(u_{l}, v\right)$ $(0 \leq l \leq L)$.

Given a consistent network of curves in space, there are an infinite number of surfaces that contain the curve network. However, a Gordon surface is a very elegant solution for the curve-surface fitting problem:

$$
\begin{aligned}
\mathrm{S}(u, v) & =\sum_{l=0}^{s} \mathrm{C}_{l}(v) \alpha_{l}(u)+\sum_{k=0}^{r} \mathrm{C}_{k}(u) \beta_{k}(v)-\sum_{l=0}^{s} \sum_{k=0}^{r} \mathrm{Q}_{l, k} \alpha_{l}(u) \beta_{k}(v) \\
& =\mathrm{S}_{1}(u, v)+\mathrm{S}_{2}(u, v)-\mathrm{T}(u, v)
\end{aligned}
$$

where $\left\{\alpha_{l}(u)\right\}_{l=0}^{s}$ and $\left\{\beta_{k}(v)\right\}_{k=0}^{r}$ are any two sets of blending functions that satisfy the constraints:

$$
\begin{aligned}
& \alpha_{l}\left(u_{i}\right)= \begin{cases}0 & \text { if } l \neq i \\
1 & \text { if } l=i\end{cases} \\
& \beta_{k}\left(v_{i}\right)= \begin{cases}0 & \text { if } k \neq i \\
1 & \text { if } k=i\end{cases}
\end{aligned}
$$

The solution can also be intended as the sum of three particular surfaces: the first $\mathrm{S}_{1}(u, v)$ and the second $\mathrm{S}_{2}(u, v)$ contain all $\mathrm{C}_{l}(v)$ and $\mathrm{C}_{k}(u)$; the third function $\mathrm{T}(u, v)$ (a tensor product) contains the intersection points between the curves.

Both $\mathrm{S}_{1}(u, v)$ and $\mathrm{S}_{2}(u, v)$ have remarkable properties, as they are skinned functions. Skinning, also termed lofting (Woodward, 1988), can be defined as the procedure to create a surface given a set of curves $\left\{\mathrm{C}_{k}(u)\right\}$ in the $u$ direction with respect to a blending direction $v$. Skinning was a very popular technique in aircraft and boat design, even before the adoption of 3D modelling software.

The second solution used in this work relies on curves that do not satisfy the previous requirements (for example, any of the curves in one direction of the network do not intersect all of the other curves). Surface deformation (Hu et al., 2001; Brujic et al., 2002) is a different strategy that provides for progressive modifications to a seed surface according to a given curve network and point cloud. In the proposed methodology, an initial geometry is progressively adjusted to transform a planar seed surface into a new 3D surface that follows the point cloud closely. In the case of Castel Masegra, both solutions were adopted. However, the vaults shown in Fig. 6 were modelled only with the second methodology, which is much faster since the (manual) generation of a consistent and detailed network is a very time-consuming process.

The chosen seed surface is a plane with a predefined number of $U$ and $V$ subdivisions (usually $32 \times 32$ ). The method exploits the property of local support of NURBS, for which a manipulation of a part of the surface provides modifications only in a confined area, without changing the whole surface. Modifications to the seed surface can be performed by 

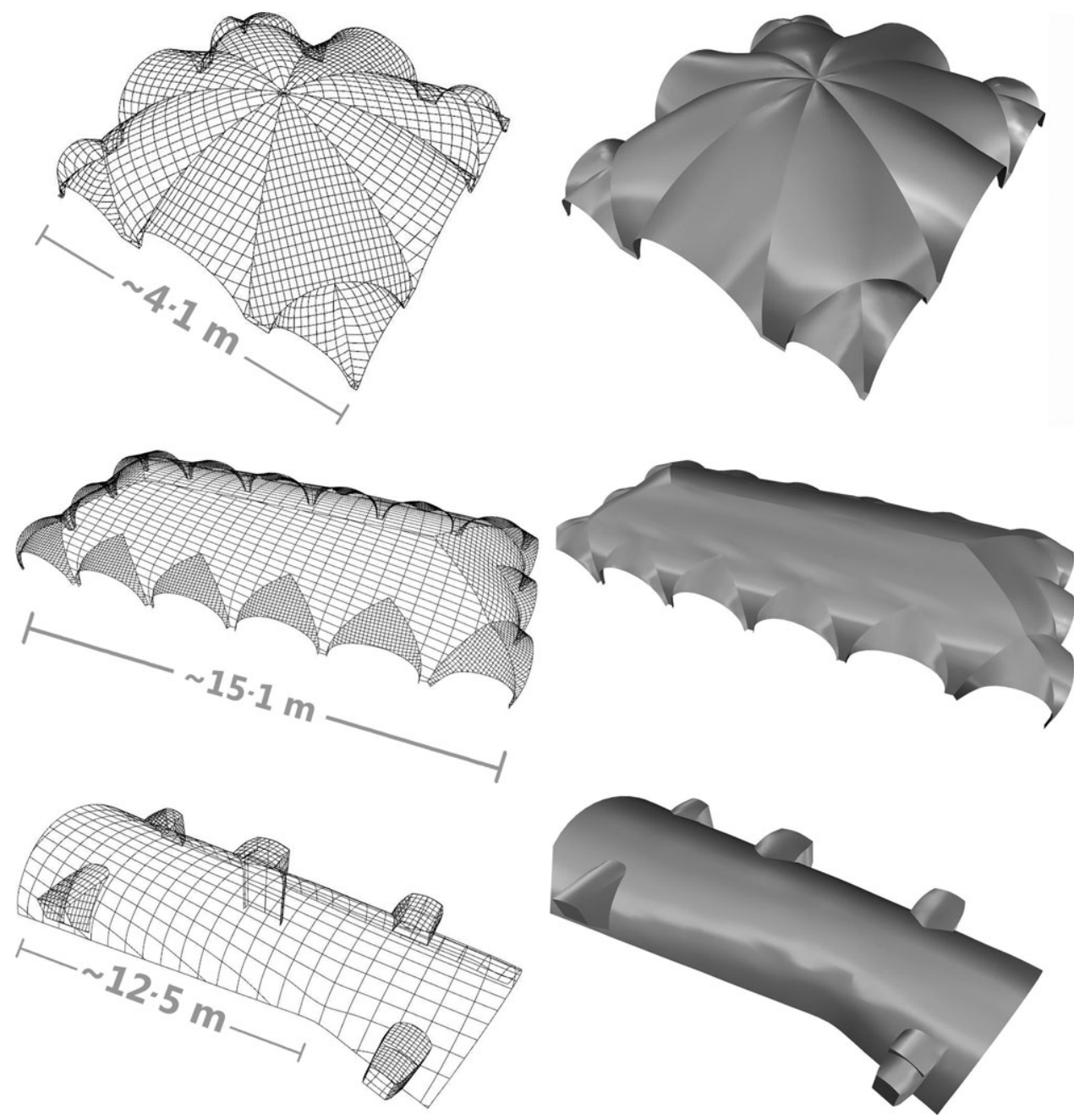

FIG. 6. The surfaces of the vaults generated with NURBS surfaces. Left: visualisation with the complete frame and its subdivision. Right: the final vaults.

modifying weights, control points and knot vectors. The procedure is carried out by considering multiple modifications not limited to a single parameter. For instance, the modification of single control points leads to an unnatural final shape, whereas altering a set of control points provides a much more realistic and smooth surface (Piegl, 1989b).

The advantages of this second strategy rely on a fully automated procedure that takes into consideration existing curves and point clouds. Another parameter that must be set beforehand is the orientation of the subdivisions, which do not follow the dominant direction of a real object in the case of fully automated processing. The main disadvantage is the smaller overall control in an interactive Gordon solution, which could result in unrealistic reconstructions for weak curve networks and non-uniform point clouds. 
Boundaries (also called discontinuity lines or edges) are also extremely important because they delineate the appearance of a freeform shape. They are used to interpolate the surface but also to combine multiple surfaces by means of trimming operations. Common boundaries for multiple surfaces (derived from the initial NURBS curves) allow a complete reconstruction without voids or intersecting surfaces.

The surfaces modelled from NURBS curves (degree 3 is always chosen for complex shapes) and point clouds are shown in Fig. 6. The NURBS surface was generated with the automatic progressive adaptation of an initial planar surface, which was generated by setting the number of internal subdivisions. Degree 3 was chosen to obtain a smooth representation with the NURBS curves used as breaklines between consecutive structural elements. This also ensures the geometric continuity of adjacent surfaces with a perfect edge-to-edge correspondence, resulting in rigorous geometric consistency of the surfaces.

Surface interpolation can be carried out with efficient algorithms that provide real-time results, allowing an immediate visual inspection of the final surfaces. The user selects the NURBS curves and the part of the point cloud used to adapt the (initial) planar NURBS surface. The solution is extremely efficient from a computational point of view so that a single surface can be fitted in less than 1 or $2 \mathrm{~s}$.

Complex objects comprise multiple parts; data processing requires the subdivision of each object depending on the discontinuity of the lines. For instance, in the case of the umbrella vault, 20 surfaces were independently fitted with the constraint of common edges (Fig. 6). This requires only the manual selection of the different parts by means of NURBS edges, whereas surface fitting is a real-time process.

As data modelling is carried out in the reference system given by the geodetic network, a set of oriented images can be automatically re-projected onto the shaded surface to obtain photo-realist visualisation (Fig. 7). A texturised 3D model is not only an attractive visual representation of the object; it also provides additional information for the generation of BIM objects by interpreting the different materials and the state of degradation. Additionally, orthophotos can be generated by re-projecting the texturised NURBS surfaces onto a plane.

A final consideration deserves to be mentioned. NURBS surfaces can be used to represent both complex shapes or predefined mathematical surfaces, such as cylinders, spheres, paraboloids and toroidal patches. In addition, they can represent ruled surfaces by means of a generatrix (a $q$ th-degree NURBS curve $\mathrm{C}(v)=\sum_{j=0}^{n} R_{j, q}(v) \mathrm{P}_{j}$ on the knot vector $V$ ) rotated around an axis. Cylinders and cones are typical examples that can be intended as surfaces of revolution, but the method becomes extremely useful when, in the case of historical objects, the generatrix can be digitised from the point clouds. This makes NURBS useful for a large variety of objects.

\section{From NURBS SURFACES TO BIM}

The final step of the proposed procedure for BIM generation concerns the conversion of the surfaces into parametric objects.

As mentioned in the previous sections, NURBS curves and surfaces are not intelligent BIM objects; hence, the 3D model made up of curves and surfaces is not a BIM. On the other hand, NURBS are unequivocally described by a finite set of parameters (degree, a set of weighted control points and a knot vector). The key idea for their BIM representation is a mathematical solution that (a) preserves the original NURBS surface and (b) adds new object-based information including semantic data and attributes. 

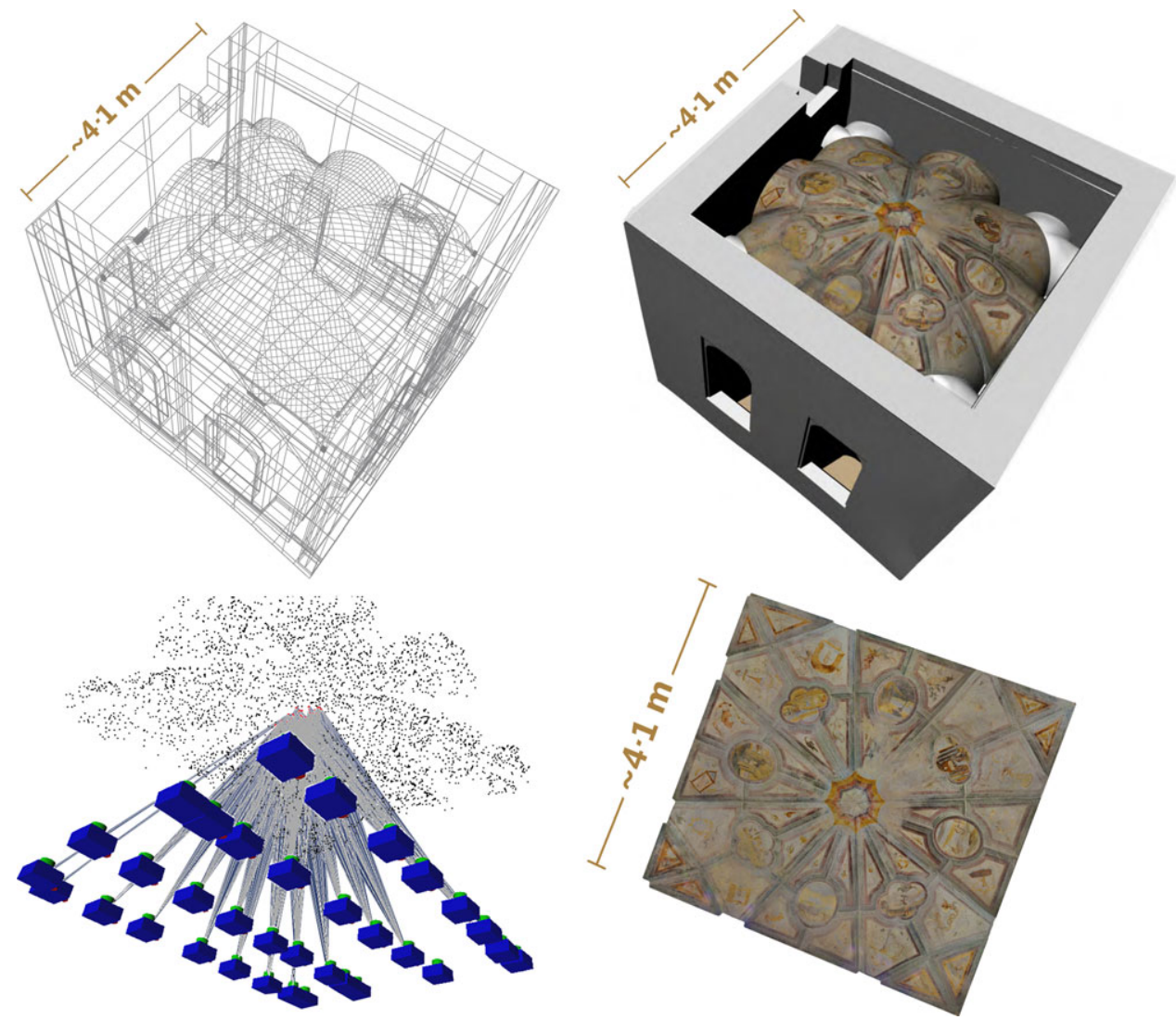

FIG. 7. Top: the NURBS surface can be textured with digital images to obtain photo-realistic visualisation. Bottom left: as images are oriented in the reference system given by total-station data, this part of the work is fully automated. Bottom right: orthophotos can be subsequently generated by re-projecting the model onto a plane.

The step towards a conversion of the surfaces into BIM objects is achieved by using both geometric and functional requirements. Generally speaking, a structural element should know what it is (for example, a wall, a staircase); it has a parametric representation of the shape together with spatial relationships with other objects. As commercial BIM packages were not developed for complex objects, there is a lack of tools for managing complex NURBS curves and surfaces in such packages.

The procedure proposed in this paper provides BIM objects of complex elements by using the NURBS surface turned into specific BIM families. This choice is motivated by the growing request for interoperable BIM projects based on different software. As families should be interoperable between most commercial software, the proposed solution provides the opportunity to work with commercial packages and makes the procedure developed useful for practical applications with various specialists who are often not very experienced in the field of metric reconstruction from point clouds.

Autodesk Revit is the BIM software package chosen in this work. In particular, Revit Architecture is the leader in the commercial market and the first software solution for direct 
link interfaces in many practical applications (Eastman et al., 2008). It has a hierarchical representation of basic objects. For instance, a column can be modelled with the available libraries starting from the category column, choosing the correct family (round column, rectangular column, and so on) and setting type information (such as the dimensions) to generate different instances. Although this procedure refers to the terminology of this specific software product, the interoperability of BIM should guarantee an efficient exchange of information between different software and tools available on the market.

The problem behind the generation of a parametric object of the complex vaults shown in the previous figures relies on the conversion of a pure 3D surface into an intelligent object. The problem can be stated as follows: given the 3D reconstruction of the intrados, which are the available object families that can provide an interoperable parametric representation?

Different default families are offered by Revit (floor, roof, stairs, ceiling and so on) and the choice should be driven by: (a) the requirements of the different specialists involved in the project; (b) the architectural elements; and (c) the final level of detail.

Vaults are one of the most complicated objects when a correct BIM representation is the final goal, especially in the case of laser-scanning point clouds that reveal geometric irregularities. A list of architectural vaults (barrel, groin, rib, fan and so on) can be found in different textbooks, and the development of global families could be used to generate a set of BIM objects. However, these objects are only "pure geometric reconstructions"; they do not highlight the complex and variable geometry captured by point clouds. In other words, they provide generic vaults where the shape is only a coarse representation when compared to $3 \mathrm{D}$ models based on point clouds.

NURBS surfaces allow the external layer to be modelled accurately, but BIM packages require a volumetric representation made up of an assembly of various layers. The wall family in Revit has the desired multilayer structure, but Revit lacks the advanced geometric modelling procedures that are able to preserve the richness of information inherent in the point cloud. For these reasons, the proposed approach uses NURBS surfaces with an associated parametric intelligence that is able to change dynamically the thickness of the vault. This is a fundamental advantage in most actual surveys where the vault thickness can only be inferred. The parametric BIM logic will then facilitate automated updating when more information is available.

The multilayer structure can be generated by multiple offsets of the NURBS surface of the intrados. Given a NURBS surface $\mathrm{S}(u, v)$ that represents a part of the vault, the offset surface is $\mathrm{S}^{\prime}(u, v)=\mathrm{S}(u, v)+\delta \mathrm{N}(u, v)$, where $\delta$ is the offset distance and $\mathrm{N}$ the normal vector. $\mathrm{S}^{\prime}(u, v)$ is not a simple translated copy of the original surface. It is another NURBS whose estimation often relies on approximations. In fact, except for a few surfaces with specific geometry (such as cylinders and spheres) that can be offset precisely, the offset surface of a complex shape is a distinct object made up of a larger number of control points and knots.

The computation of $\mathrm{S}^{\prime}(u, v)$ can be carried out with preliminary sampling of the surface based on the second derivative (Filip et al., 1986); then, the offset surface is simplified via knot removal. The edges of the offset and original surfaces are then connected with linear features to obtain a volumetric representation.

The results for the vault in the reception hall of Castel Masegra are shown in Fig. 8. The red/brown part is already a parametric object with an initial thickness set by the operator and with a multilayer structure. The selected blue part was parametrised and the materials set in Revit. The black parts remain as non-parameterised surfaces. 
The vaults generated with the proposed method and then integrated in Autodesk Revit are shown in Fig. 9. Vaults are considered complete BIM objects by the software, therefore size, material properties and layers can be modified in the software's command bar.

As the computation of NURBS surfaces involves approximations, some problems were found in the case of complex objects. The layer-based reconstruction from the intrados to the extrados is not a simple extrusion. It involves a large number of offset directions in space depending on the surface curvature. Some local intrusions or a lack of edge correspondence were found for consecutive surfaces, which are precisely joined only on the intrados and external boundaries. This means that geometric continuity is not guaranteed for the whole layer structure, and future work will be essential to correct this effect. On the other hand, the problem can be found only when no information about the extrados is available; otherwise, a second NURBS surface could be generated without parameterisation of the thickness. Because NURBS are quite smooth surfaces, the impact of the rigorous estimation of the final volume with or without intrusion effects is surely a minor error with a small influence in practical applications (for example, for an estimate of the cost).

\section{Accuracy Evaluation and Data Processing Time}

In the case of complex objects with irregular shapes, an additional quality check of the NURBS surface is recommended. A simple inspection can rapidly be carried out by

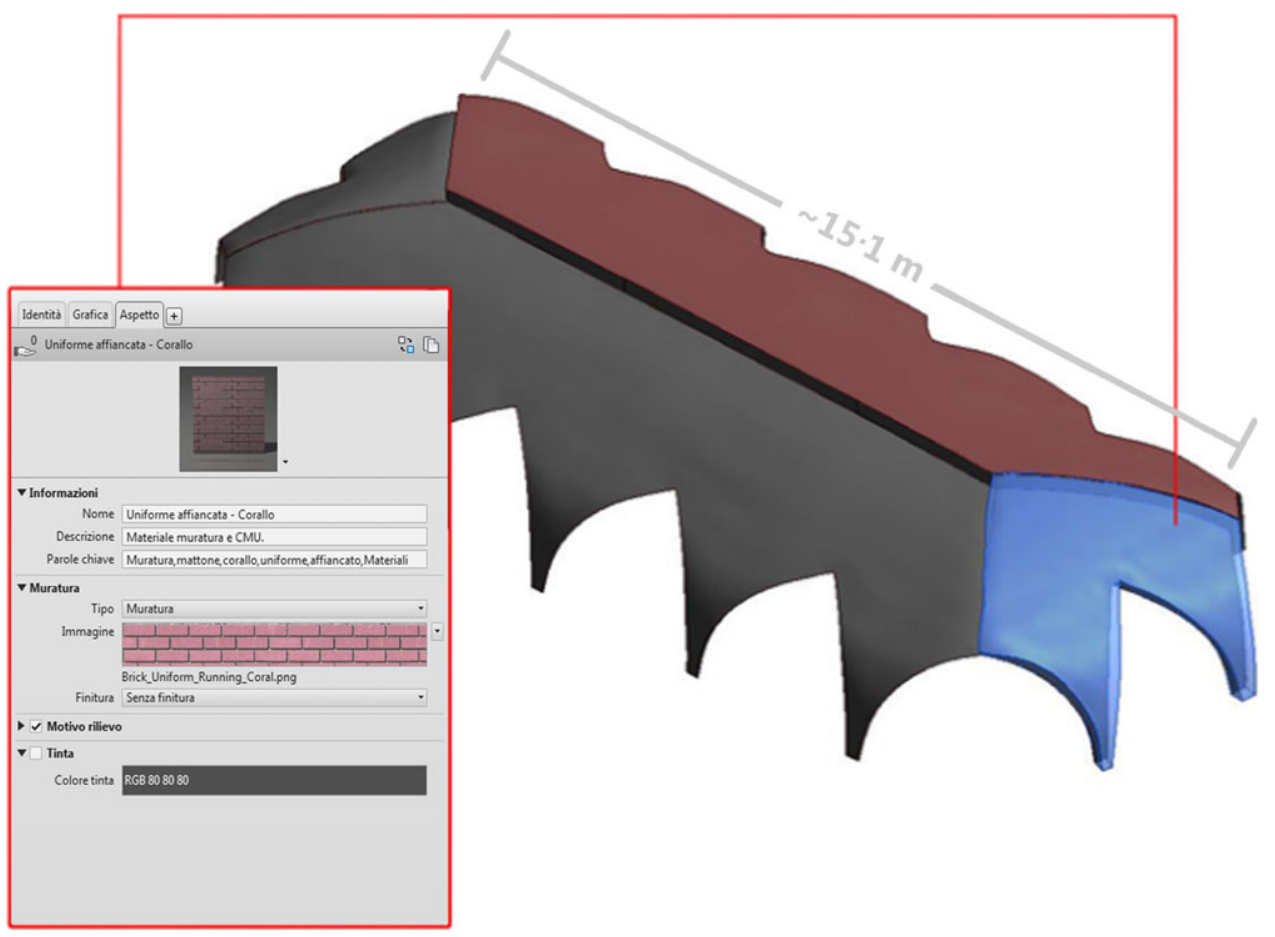

FIG. 8. The vaults become parametric BIM objects in which the thickness can be interactively set to obtain a volumetric representation. 
BARAZZETTI et al. Creation of parametric BIM objects from point clouds using NURBS
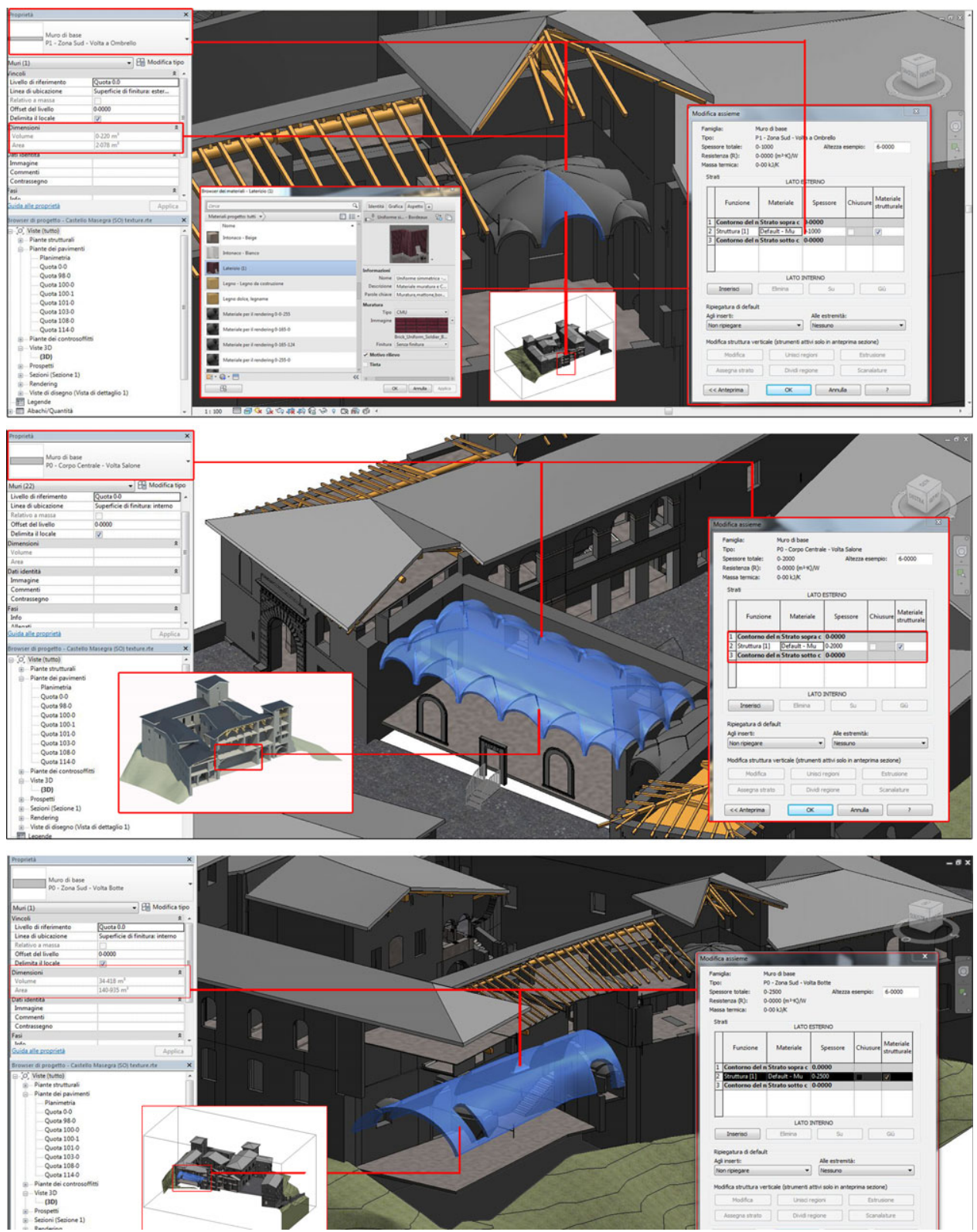

FIG. 9. The vaults can be imported in BIM software (Autodesk Revit in this case) and their size and properties (materials, layers and so on) can be interactively modified.

using the point cloud to estimate the overall geometric discrepancy and error distribution. Fig. 10 shows the accuracy evaluation for the vault of the reception hall. The root mean square (RMS) between the cloud and the NURBS surface is $0.003 \mathrm{~m}$ (estimated with 


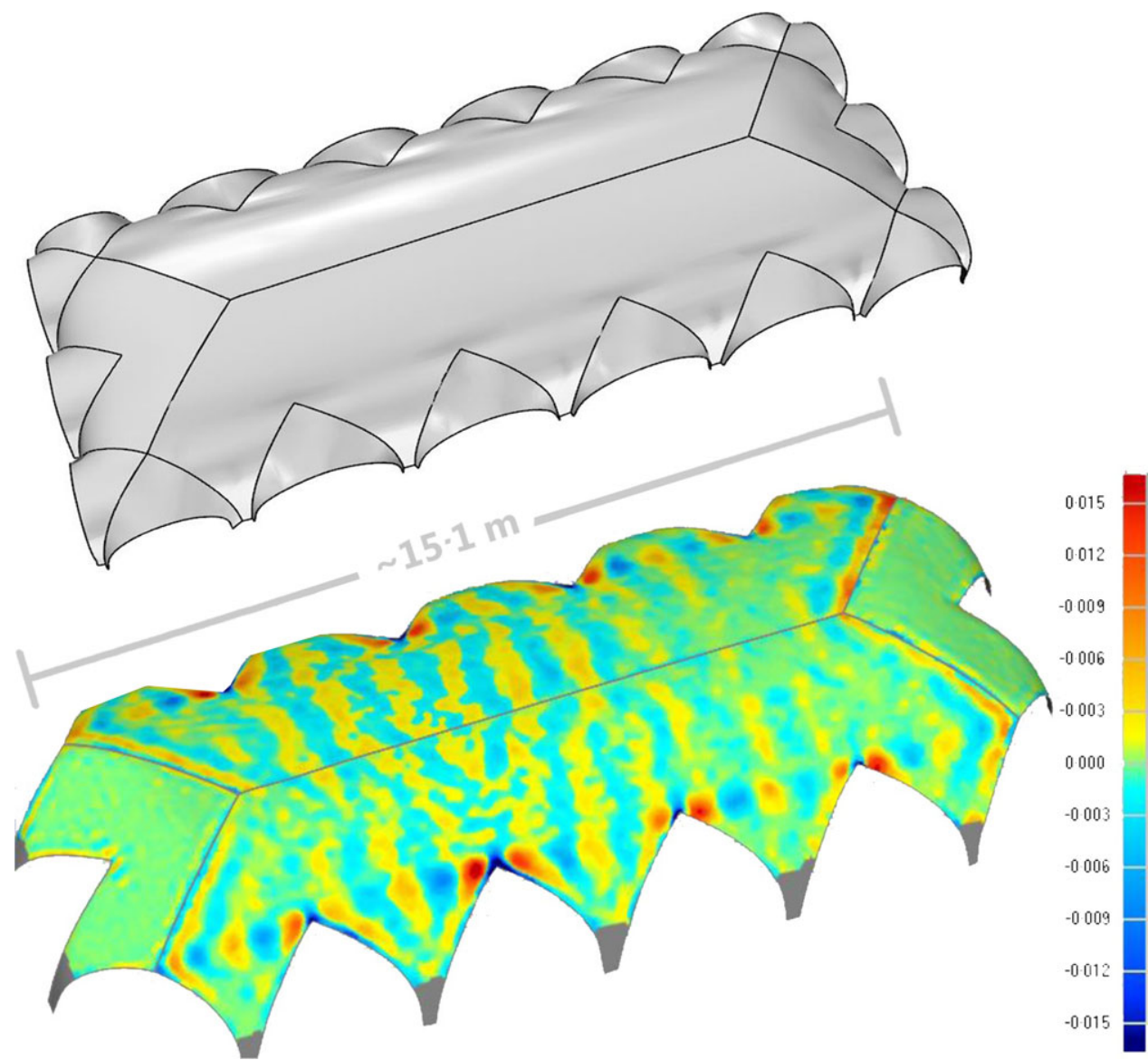

FIG. 10. An irregular vault scanned with 22 million points and a comparison with the final NURBS surface along the normal direction after the generation of the solution. Dark red and dark blue indicate the largest differences. Statistics shows an average discrepancy of $0.003 \mathrm{~m}$ in terms of RMS.

Geomagic Studio) and the vault is about $15 \mathrm{~m} \times 6.5 \mathrm{~m} \times 2 \mathrm{~m}$. The laser scanner used was a Faro Focus 3D with a nominal precision of about $\pm 0.002 \mathrm{~m}$. Therefore, the analysis shows a discrepancy very similar to the precision of the laser scanner. As similar results were found for the other vaults, the use of NURBS surfaces was an efficient solution for reconstructing these kinds of objects.

The vaults presented in this paper were generated with a set of default parameters for the different algorithms, which were then tested in order to find values that were suitable for most applications. The default parameters usually remain constant for the different parts of structural elements, notwithstanding the level of detail which depends on instrument precision and scale of representation. This ensures fast data processing during the estimation of curves and surface parameters which results in global processing times that are considered acceptable for most practical purposes. In particular, after several 
experiments, algorithm parameters for the reconstruction of the curves and surfaces were set as follows:

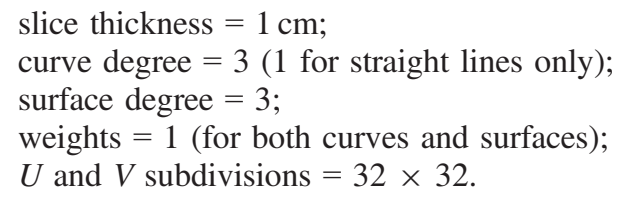

The choice of these parameters depends on metric accuracy, processing time and available memory. The non-excessive size of the final NURBS file is a fundamental property in order to operate with BIM packages that were not developed for complex shapes. The final result for the vault surface in Fig. 9 consists of NURBS surfaces saved in CAD (.dwg) format. Memory size is only $160 \mathrm{kB}$ and allows a rigorous mathematical representation from a point cloud made up of 22 million points. This means that a very small memory is required to store NURBS surfaces. Complex sites made up of several objects can be digitally reconstructed with the proposed methodology. Other file formats that are able to store NURBS precisely were not taken into consideration because of their larger memory requirements (for example, STEP uses $420 \mathrm{kB}$ and IGES occupies $540 \mathrm{kB}$ ). The parametric BIM model of the vault is $2 \mathrm{MB}$ in .rte (Revit) BIM format.

Data processing time greatly depends on the manual identification of discontinuity lines for the separation of structural elements, whereas the time for the computation of the NURBS surface is much shorter. In the case of the vault in Fig. 10, global processing time can be subdivided as follows:

(1) manual generation of discontinuity lines with NURBS curves: $40 \mathrm{~min}$;

(2) automated generation of the NURBS surfaces, including curves as boundaries and the point cloud as constraint for interpolation: less than $5 \mathrm{~s}$;

(3) geometric parametrisation of the thickness with the multilayer object: processing time less than $2 \mathrm{~s}$ (the user must only select the object and assign a specific family);

(4) inclusion of attributes: this part depends on the information available for each specific object (such as materials, thermal properties).

The computer used was a workstation with an Intel ${ }^{\circledR}$ Xeon ${ }^{\circledR}$ CPU E5-2609 - $2.4 \mathrm{GHz}$, with 32 GB of RAM and a Quadro 2000 graphic card. Similar results were found for the different vaults shown in the paper. This means that the first part of the work, namely, the manual identification of discontinuity lines, is more time consuming and depends on the ability of the operator both to identify the different structural components and also model its boundaries. Overall, it is not simple to provide general criteria that assess the required time of this part of the reconstruction process, since it is strongly dependent on the morphology of the object.

Much research work is required in this first step. As matters currently stand, automated object recognition and machine-vision algorithms are still not able to separate the different architectural components surveyed with noisy point clouds.

\section{CONCLUSIONS}

This paper has presented a procedure for BIM generation from point clouds via BIM parametrisation of NURBS curves and surfaces. The particular case of complex architecture was addressed in order to cope with the lack of commercial BIM software able to handle irregular freeform shapes. 
The proposed methodology is able to preserve the level of detail in the geometric information provided by photogrammetric and laser-scanner point clouds. The 3D model of the surfaces is then converted into multiple parametric BIM objects with parametric intelligence, relationships to other objects and attributes. The procedure is intended to be a semi-automated approach where the expert operator investigates the building to separate its different structural elements. NURBS allows the reconstruction of the geometric complexity of irregular elements following the (manually) extracted discontinuity lines in space, which can be then densified with an additional curve network anchored to the point cloud. NURBS surfaces are then generated with an automated interpolation.

NURBS were chosen to initialise the reconstruction because simple shapes and complex forms can be accurately modelled. NURBS are rigorous mathematical functions that can be modified, copied and stored with limited computational cost and memory requirements. Moreover, they can reconstruct simple geometric shapes as well as irregular forms. The mathematical representation of NURBS curves and surfaces is then used to achieve BIM parametrisation. This provides the BIM database along with the opportunity to add material properties.

As things stand at the present, the commercial market offers some sophisticated NURBS-based modellers that are not BIM software, whereas BIM packages are mainly designed for modern construction projects with simple geometric forms. The generation of advanced BIM models from point clouds is still a very complicated task in the case of complex shapes. The proposed semi-automated procedure is a new efficient solution which has been tested in real projects.

It is evident that the current interest in BIM technology is increasing the demand for digital representation techniques in the construction industry. Photogrammetry and laser scanning can make a remarkable contribution to the development of interoperable BIM, where the geometric component must be integrated with the logic of construction. This task will require new advanced manual, semi-automatic and fully automated solutions to understand and schematise the structure and its behaviour.

\section{REFERENCES}

ArAyci, Y., 2008. Towards building information modelling for existing structures. Structural Survey, 26(3): 210-222.

AzHAR, S., 2011. Building information modeling (BIM): trends, benefits, risks and challenges for the AEC industry. ASCE Journal of Leadership and Management in Engineering, 11(3): 241-252.

Azhar, S., Khalfan, M. and Maqsood, T., 2012. Building information modeling (BIM): now and beyond. Australasian Journal of Construction Economics and Building, 12(4): 15-28.

Baik, A., Alitany, A., Boenm, J. and Robson, S., 2014. Jeddah historical building information modelling "JHBIM" - object library. ISPRS Annals of Photogrammetry, Remote Sensing and Spatial Information Sciences, 2(5): 41-47.

Barazzetti, L., Scaioni, M. and Remondino, F., 2010. Orientation and 3D modelling from markerless terrestrial images: combining accuracy with automation. Photogrammetric Record, 25(132): 356-381.

Barazzetti, L., Brumana, R., Oreni, D., Previtali, M. and Roncoroni, F., 2014. UAV-based orthophoto generation in urban area: the Basilica of Santa Maria di Collemaggio in L'Aquila. Lecture Notes in Computer Science, 8582: 1-13.

Barazzetti, L., Banfi, F., Brumana, R., Gusmeroli, G., Oreni, D., Previtali, M., Roncoroni, F. and Schiantarelli, G., 2015. BIM from laser clouds and finite element analysis: combining structural analysis and geometric complexity. International Archives of Photogrammetry, Remote Sensing and Spatial Information Sciences, 40(5/W4): 345-350.

Brujic, D., Ristic, M. and Ainsworth, I., 2002. Measurement-based modification of NURBS surfaces. Computer-Aided Design, 34(3): 173-183. 
BARAZZETTI et al. Creation of parametric BIM objects from point clouds using NURBS

Brumana, R., Oreni, D., Raimondi, A., Georgopoulos, A. and Breggiani, A., 2013. From survey to HBIM for documentation, dissemination and management of built heritage: the case study of St. Maria in Scaria d'Intelvi. Digital Heritage International Congress, Marseille, France. 1: 497-504.

Cabaleiro, M., Riveiro, B., Arias, P., Caamaño, J. C. and Vilán, J. A., 2014. Automatic 3D modelling of metal frame connections from LiDAR data for structural engineering purposes. ISPRS Journal of Photogrammetry and Remote Sensing, 96: 47-56.

Chelson, D. E., 2010. The effects of building information modeling on construction site productivity. $\mathrm{PhD}$ thesis, University of Maryland, USA. 314 pages.

Cox, M. G., 1972. The numerical evaluation of B-splines. IMA Journal of Applied Mathematics, 10(2): 134149.

Cronk, S., Fraser, C. and Hanley, H., 2006. Automated metric calibration of colour digital cameras. Photogrammetric Record, 21(116): 355-372.

Dore, C., Murphy, M., McCarthy, S., Brechin, F., Casidy, C. and Dirix, E., 2015. Structural simulations and conservation analysis - historic building information model (HBIM). International Archives of Photogrammetry, Remote Sensing and Spatial Information Sciences, 40(5/W4): 351-357.

Eastman, C., Teicholz, P., Sacks, R. and Liston, K., 2008. BIM Handbook: A Guide to Building Information Modeling. Wiley, Hoboken, New Jersey, USA. 648 pages.

Fai, S., Graham, K., Duckworth, T., Wood, N. and Attar, R., 2011. Building information modelling and heritage documentation. 23rd CIPA Symposium, Prague, Czech Republic. 8 pages.

Fai, S., FilipPi, M. and Paliaga, S., 2013. Parametric modelling (BIM) for the documentation of vernacular construction methods: a BIM model for the Commissariat Building, Ottawa, Canada. ISPRS Annals of Photogrammetry, Remote Sensing and Spatial Information Sciences, 2(5/W1): 115-120.

FAI, S. and Rafeiro, J., 2014. Establishing an appropriate level of detail (LoD) for a building information model (BIM) - West Block, Parliament Hill, Ottawa, Canada. ISPRS Annals of the Photogrammetry, Remote Sensing and Spatial Information Sciences, 2(5): 123-130.

FARIN, G., 1992. From conics to NURBS: a tutorial and survey. IEEE Computer Graphics and Applications, 12(5): 78-86.

FARIN, G., 1996. Curves and Surfaces for Computer Aided Geometric Design - A Practical Guide. Fourth edition. Academic Press, Boston, Massachusetts, USA. 429 pages.

Fassi, F., Achille, C. and Fregonese, L., 2011. Surveying and modelling the main spire of Milan Cathedral using multiple data sources. Photogrammetric Record, 26(136): 462-487.

Filip, D., Magedson, R. and Markot, R., 1986. Surface algorithms using bounds on derivatives. Computer Aided Geometric Design, 3(4): 295-311.

Gordon, W. J., 1993. Sculptured surface definition via blending function methods. Fundamental Developments of Computer Aided Geometric Modeling, Academic Press, London, UK. 414 pages: 117-134.

Gruen, A., 2012. Development and status of image matching in photogrammetry. Photogrammetric Record, 27(137): 36-57.

Guidi, G., Remondino, F., Russo, M., Menna, F., Rizzi, A. and Ercoli, S., 2009. A multi-resolution methodology for the 3D modeling of large and complex archaeological areas. International Journal of Architectural Computing, 7(1): 39-55.

Hichri, N., Stefani, C., De Luca, L. and Veron, P., 2013. Review of the "as-built BIM" approaches. International Archives of Photogrammetry, Remote Sensing and Spatial Information Sciences, 40(5/W1): $107-112$.

Hsu, W. M., Hughes, J. F. and Kaufman, H., 1992. Direct manipulation of free-form deformations. Computer Graphics, 26(2): 177-184.

Hu, S.-M., Li, Y.-F., Ju, T. and ZHU, X., 2001. Modifying the shape of NURBS surfaces with geometric constraints. Computer-Aided Design, 33(12): 903-912.

JAZAYERI, I. and FRASER, C. S., 2010. Interest operators for feature-based matching in close range photogrammetry. Photogrammetric Record, 25(129): 24-41.

Kensex, K. and Noble, D., 2014. Building Information Modeling: BIM in Current and Future Practice. Wiley, Hoboken, New Jersey, USA. 432 pages.

Lerma, J. L., Navarro, S., Segui, A. E. and Cabrelles, M., 2014. Range-based versus automated markerless image-based techniques for rock art documentation. Photogrammetric Record, 29(145): 3048.

Murphy, M., McGovern, E. and Pavia, S., 2009. Historic building information modelling (HBIM). Structural Survey, 27(4): 311-327.

Murphy, M., McGovern, E. and Pavia, S., 2013. Historic building information modelling - adding intelligence to laser and image based surveys of European classical architecture. ISPRS Journal of Photogrammetry and Remote Sensing, 76: 89-102. 
Oreni, D., Brumana, R. and CucA, B., 2012. Towards a methodology for 3D content models: the reconstruction of ancient vaults for maintenance and structural behaviour in the logic of BIM management. 18th International Conference on Virtual Systems and Multimedia, Milan, Italy. 475-482.

Oreni, D., Brumana, R., Banfi, F., Bertola, L., Barazzetti, L., Cuca., B., Previtali, M. and Roncoroni, F., 2014. Beyond crude 3D models: from point clouds to historical building information modeling via NURBS. Lecture Notes in Computer Science, 8740: 166-175.

PIEGL, L., 1989a. Modifying the shape of rational B-spline. Part 1: curves. Computer-Aided Design, 21(8): 509-158.

PIEGL, L., 1989b. Modifying the shape of rational B-spline. Part 2: surfaces. Computer-Aided Design, 21(9): 538-546.

PIEGL, L., 1991. On NURBS: a survey. IEEE Computer Graphics and Applications, 11(1): 55-71.

Piegl, L. and Tiller, W., 1997. The NURBS Book. Monographs in Visual Communication. Second edition. Springer, New York, USA. 646 pages.

Quattrini, R., Malinverni, E. S., Clini, P., Nespeca, R. and Orlietti, E., 2015. From TlS to HBIM: high quality semantically-aware 3D modeling of complex architecture. International Archives of Photogrammetry, Remote Sensing and Spatial Information Sciences, 40(5/W4): 367-374.

Remondino, F., 2011. Heritage recording and 3D modeling with photogrammetry and 3D scanning. Remote Sensing, 3(6): 1104-1138.

Remondino, F. and El-Hakim, S., 2006. Image-based 3D modelling: a review. Photogrammetric Record, 21(115): 269-291.

Remondino, F., Spera, M. G., Nocerino, E., Menna, F. and Nex, F., 2014. State of the art in high density image matching. Photogrammetric Record, 29(146): 144-166.

Riveiro, B., Arias, P., Armesto, J., Rial, F. and Solla, M., 2008. Multidisciplinary approach to historic arch bridges documentation. International Archives of Photogrammetry, Remote Sensing and Spatial Information Sciences, 37(B5): 247-252.

Scianna, A., Gristina, S. and Paliaga, S., 2014. Experimental BIM applications in archaeology: a workflow. Lecture Notes in Computer Science, 8740: 490-498.

Woodward, C. D., 1988. Skinning techniques for interactive B-spline surface interpolation. Computer-Aided Design, 20(8): 441-451.

YeH, S.-S. and Hsu, P.-L., 2002. Adaptive-feedrate interpolation for parametric curves with a confined chord error. Computer-Aided Design, 34(3): 229-237.

\section{Résumé}

Cet article présente une procédure innovante pour la création d'objets paramétriques de BIM (modèle d'information de bâtiment) à partir de nuages de points sur des formes architecturales complexes. La technologie BIM exige une représentation paramétrique avancée de la géométrie comprenant des relations spatiales, des contraintes et les propriétés des matériaux. Cette procédure utilise une méthodologie de reconstruction basée sur le BIM qui préserve le niveau de détail contenu dans des nuages de points photogrammétriques ou lasergrammétriques, et qui s'appuie sur des B-splines rationnelles non uniformes (courbes NURBS). Le cas particulier d'objets architecturaux présentant des formes irrégulières est étudié car il n'existe pas de logiciels commerciaux de BIM capables de prendre en compte de tels bâtiments. Une étude de cas est menée sur un ensemble de 7,5 milliards de points pour démontrer l'utilité de la procédure proposée sur de gigantesques nuages de points.

\section{Zusammenfassung}

Dieser Beitrag präsentiert eine innovative Prozedur zur Generierung parametrischer Gebäudeobjekte als Gebäudeinformationsmodelle (BIM) aus Punktwolken von komplexen architektonischen Merkmalen. Die BIM Technologie erfordert eine hochentwickelte parametrische Repräsentation der Geometrie unter Einbeziehung räumlicher Relationen, Bedingungen und Materialeigenschaften. Das Ziel der Prozedur ist der Erhalt des Level-ofDetail, der in Punktwolken aus Photogrammetrie und Laserscanning enthalten ist und setzt daher auf nicht- 
BARAZZETTI et al. Creation of parametric BIM objects from point clouds using NURBS

uniforme rationale B-Splines (NURBS) Kurven. Da kommerzielle BIM Software Architekturobjekte mit irregulären Formen nicht beherrscht, wird hier speziell dieser Fall behandelt. An einem aktuellem Fallbeispiel mit bis zu 7.5 Milliarden Punkten wird die Anwendung der vorgeschlagenen Prozedur diskutiert.

\section{Resumen}

Este trabajo presenta un procedimiento innovador para crear objetos modelados con información parametrizada de edificios (BIM) a partir de nubes de puntos de características arquitectónicas complejas. La tecnología BIM requiere una representación paramétrica avanzada de la geometría que involucra las relaciones espaciales, las limitaciones y las propiedades del material. El objetivo del procedimiento es una metodología de reconstrucción avanzada basada en BIM que preserva el nivel de detalle encapsulado en nubes de puntos fotogramétricos y láser de barrido, y basado en NURBS avanzados. Se aborda el caso particular de objetos arquitectónicos con formas irregulares debido a la falta de software BIM comercial capaz de manejar tales edificios. Se estudia un caso real compuesto por 7,5 billones de puntos para demostrar el procedimiento propuesto con conjuntos de datos de nubes de gran número de puntos.

\section{摘要}

本文提出了一种从复杂建筑点云数据提取参数化建筑信息模型(BIM)对象的创新算法。BIM技术要求 对涉及空间关系、约束条件和材质属性等进行参数化表达。该算法是一种依赖于NURBS曲线的BIM重建 方法,可表征从摄影测量点云和激光扫描点云提取的建筑物细节特征。现在无商业化的BIM软件能提取不 规则建筑的建筑信息模型对象,采用本文提出的算法,对有75亿点云数据进行建筑信息模型对象的提取。 